ROCZNIKI HUMANISTYCZNE

Tom LXVIII, zeszyt 8 - 2020

DOI: http://dx.doi.org/10.18290/rh.20688-7

\title{
LA TRADUCTION LITTÉRAIRE COMME OUTIL DIDACTIQUE DANS L'ENSEIGNEMENT DES LANGUES ÉTRANGÈRES ? ÉTUDE DE CAS : PRZED SKLEPEM JUBILERA DE KAROL WOJTYŁA EN TRADUCTION L2
}

\author{
LITERARY TRANSLATION AS A TEACHING TOOL \\ IN FOREIGN LANGUAGE TEACHING? \\ CASE STUDY: L2-TRANSLATION OF PRZED SKLEPEM JUBILERA \\ BY KAROL WOJTYŁA
}

\section{Abstract}

In recent years there has been a reassessment of the role of translation and translations in foreign language teaching and education (Cook; Laviosa; Carreres, "Translation »). In 2001 the Common European Framework of Reference (CEFR) recognized translation and interpretation as a language activity which is called mediation. Literary translation is being proposed as a mediation activity intended to develop the (written) mediation competence of foreign language learners. This article reports on the results of a case study in which we examine to what extend the L2 translation of drama indeed does enhance mediation skills of language learners. In this case study, we decided to investigate the extent to which Dutch language learners apply mediation strategies when translating an excerpt from Przed sklepem jubilera (The Jeweler's Shop) (written by Karol Wojtyła) in Dutch (L2), and the extent to which their strategies differ from those we found in the (official) Dutch translation of the play.

Key words: translation didactics; pedagogical translation; mediation; translation strategies; literary translation.

Docteur hab. Muriel Waterlot - Chaire de linguistique néerlandaise de l'Université Catholique Jean Paul II de Lublin (Pologne); adresse postale : Katedra Języka Niderlandzkiego IFA, Katolicki Uniwersytet Jana Pawła II, Al. Racławickie 14, 20-950 Lublin, Pologne ; e-mail : murielwaterlot@kul.pl. ORCID: https://orcid.org/0000-0002-1665-2543.

Dr hab. Muriel Waterlot - Katolicki Uniwersytet Jana Pawła II, Wydział Nauk Humanistycznych, Instytut Językoznawstwa, Katedra Języka Niderlandzkiego; adres do korespondencji: Katedra Języka Niderlandzkiego IFA KUL, Al. Racławickie 14, 20-950 Lublin; e-mail : murielwaterlot@kul.pl. ORCID: https://orcid.org/0000-0002-1665-2543. 
Ces dernières années, le rôle de la traduction et des traductions dans l'enseignement des langues étrangères a été réévalué (Cook ; Laviosa ; Carreres, «Translation »). En 2001, le Cadre européen commun de référence (CECR) a reconnu la traduction et l'interprétation comme une activité linguistique appelée médiation. La traduction littéraire est proposée comme une activité de médiation, destinée à améliorer la compétence de médiation écrite des apprenants en langues étrangères.

Cet article rend compte des résultats d'une étude de cas dans laquelle nous examinons dans quelle mesure la traduction de pièces de théâtre en L2 améliore l'aptitude en matière de médiation des apprenants du néerlandais langue étrangère à l'Université Catholique de Lublin Jean-Paul II de Lublin (KUL). Dans les premiers paragraphes, nous passons en revue la situation de la traduction dans l'enseignement des langues étrangères (e.a. les pour et les contre). Nous présentons aussi des stratégies de traduction selon Andrew Chesterman qui seront illustrées par les passages de la version polonaise et néerlandaise de la pièce Przed sklepem Jubilera (fr. La boutique de l'orphèvre) de Karol Wojtyla. Dans le troisième paragraphe, nous présentons l'objectif, l'hypothèse et la méthodologie d'une étude de cas dans laquelle nous analysons la mesure dans laquelle les apprenants avancés polonais du néerlandais langue étrangère de la KUL utilisent des stratégies de médiation dans la traduction d'un texte littéraire en L2. Dans le quatrième paragraphe nous présentons les résultats de notre analyse.

\section{REVALORISATION DE LA TRADUCTION DANS L'ENSEIGNEMENT DES LANGUES ÉTRANGÈRES}

À la fin du XIX ${ }^{\mathrm{e}}$ siècle, avec l'avènement de la méthode directe (cf. ang. Direct Method) ou la méthode naturelle (cf. ang. Natural Method), la traduction a été supprimée des cours de langue (Howatt 192). En effet, les opposants à la méthode grammaire et traduction l'ont considérée comme contre-productive dans le processus d'acquisition d'une langue, notamment pour le développement des capacités d'expression orale. Influencée par l'internationalisation de la société et l'intérêt croissant pour la dimension communicative interculturelle dans l'enseignement des langues étrangères, la traduction a été revalorisée depuis la mi-1990, et depuis 2001 même réintroduite sous le nom de médiation (ang. mediation) par le Cadre Européen Commun de Référence pour les Langues (CECR). Parmi les types d'activités 
de médiation, le CECR inclut la traduction et l'interprétation (CECR [2001] 147). Cela signifie qu'aujourd'hui la recherche sur la traduction en tant que méthode de travail ne se concentre plus sur son efficacité dans l'enseignement des langues étrangères (= le pour et le contre), mais sur la manière dont elle peut être appliquée dans les cours d'acquisition des langues (= l'approche didactique). Selon le CECR, la traduction peut en particulier être utilisée comme un outil didactique pour promouvoir des compétences spécifiques, telles que les compétences générales et communicatives des apprenants en langues (CECR [2018] 13, 98). Cependant, l'absence de descripteurs de cette activité linguistique dans le CECR a conduit à ce que les activités dites de médiation soient toujours négligées dans les cours de langue. En 2017 le Conseil de l'Europe a finalement publié un supplément au CECR avec de nouveaux descripteurs et échelles de notation pour les activités de médiation. La traduction en français de ce document intitulée Volume complémentaire du Cadre européen commun de référence pour les langues : apprendre, enseigner, évaluer est apparue en 2018 (CECR [2018]).

Selon le CECR, la traduction écrite et orale d'un texte relève de la médiation des textes qui signifie "transmettre à une personne le contenu d'un texte auquel cette personne n'aurait pas accès, souvent à cause de barrières d'ordre linguistique, culturel, sémantique ou technique. C'est là le sens principal donné au terme de médiation dans le texte du CECR 2001" (CECR [2018] 109). Cette description nous montre que dans les activités de médiation, le contenu l'emporte sur la mise en forme du message.

Pour réaliser des activités de médiation, le Volume complémentaire du CECR propose des «stratégies de médiation » définies comme suit:

Les stratégies de médiation sont des techniques utilisées pour clarifier le sens de ce qui est dit ou écrit et faciliter la compréhension. (...) Elles portent sur la façon de traiter un contenu original à l'intention du destinataire. Est-il par exemple nécessaire de développer ce contenu, le condenser, le paraphraser, le simplifier, l'illustrer avec des métaphores ou des documents visuels? Les stratégies sont présentées l'une après l'autre parce qu'elles s'appliquent à de nombreuses activités. (CECR [2018] 132; caractères gras M.W.)

Nous pouvons en déduire que des stratégies telles que la paraphrase, la simplification d'un texte ou l'ajout d'informations supplémentaires pour rendre un texte plus compréhensible peuvent être utilisées par un médiateur (=1'apprenant) pour favoriser la compréhension du texte.

En outre le Volume complémentaire fait mention de «stratégies pour simplifier un texte » en vue d'une meilleure compréhension du texte. Cela peut 
impliquer d'une part l'amplification d'un texte dense par l'ajout d'informations utiles, d'exemples, de détails, d'informations de base, de commentaires raisonnés et explicatifs (CECR [2018] 133). D'autre part cela peut également signifier d'élaguer un texte. Cela peut consister à restituer une information en moins de mots après avoir éliminé les répétitions et les digressions, et à supprimer les parties du texte source qui n'apportent pas d'information pertinente nouvelle (CECR [2018]133).

$\mathrm{Vu}$ que la traduction fait partie des activités de médiation, nous pouvons en principe admettre que dans le transfert d'un texte d'une langue vers une autre, le traducteur aura recours aux mêmes genres de stratégies. Nous reviendrons sur ce point lors de la définition de l'hypothèse de recherche (cf. 3.1.).

En ce qui concerne les activités de médiation écrite nous trouvons dans le CERC [2001] (71) les exemples suivants:

- traduction précise (de contrats, de textes de loi, de textes scientifiques, etc.),

- traduction littéraire (romans, théâtre, poésie, livrets, etc.),

- résumé de l'essentiel (articles de journaux et magazines, etc.) en L2 ou entre $\mathrm{L} 1$ et $\mathrm{L} 2$,

- reformulation (textes spécialisés pour non spécialistes, etc.).

Ainsi, selon le CECR, la traduction de pièces de théâtre serait appropriée pour le développement des compétences de médiation des apprenants. Nous examinerons, sur la base d'une étude de cas, si c'est effectivement le cas pour la traduction en L2.

\section{LA TRADUCTION LITTÉRAIRE EN L2 DANS L'ENSEIGNEMENT DES LANGUES ÉTRANGÈRES}

Dans la littérature sur la (ré)intégration de la traduction dans l'enseignement des langues, l'attention est principalement portée sur la recherche sur la traduction en L1. Dans certaines études, nous trouvons des indices concernant la traduction en L2. Dans ce contexte nous pouvons citer comme exemples les travaux de Duff, Cook et Carreres («Strange Bedfellows» 3). Dans "Strange bedfellows: Translation and Language teaching. The teaching of translation into L2 in modern languages degrees; uses and limitations » Carreres aborde en détail le débat sur la traduction en L2 en classe d'acquisition des langues étrangères. Elle indique que l'enseignement de la traduction en L2 a été principalement critiqué à cause du fait que la 
traduction en L2 est irréaliste (en principe les traducteurs traduisent de leur L2 et L3 vers leur L1 (voir les dispositions de la Direction générale de la traduction de l'Union européenne pour les traducteurs) et donc complètement inutile (Carreres, "Strange Bedfellows", "Translation»). Dans son article, elle se demande néanmoins pourquoi les universitaires ne l'interdisent pas définitivement dans le programme d'études :

Indeed, one may wonder, if academics are so convinced that L2 translation is, in short, a bad idea, why are they stoically writing papers on how to best weather the storm, instead of trying to banish it from the curriculum? (Carreres, «Strange Bedfellows 》4)

Un des avantages de la traduction littéraire réside dans le fait que les apprenants sont en mesure de se faire une idée des différences linguistiques à quatre niveaux différents: le niveau des différences sémantiques (sens des mots), les différences syntaxiques, les différences de style (formulation, imagerie, phraséologie) et le niveau de cohésion du texte (Missine 411-418). Bien entendu, les traductions en L2 ne concernent pas le résultat, le produit final, ni la traduction parfaite, mais 'l'effort de traduction', le processus de traduction. On peut ajouter qu'en traduisant des textes de théâtre ou de poésie les élèves sont plus enclins à la discussion car, en vue de préserver le sens et la forme du texte, le traducteur peut être contraint à avoir recours à une plus grande liberté de traduction.

En ce qui concerne les inconvénients, nous devrons bien sûr tenir compte du fait que non pas tous les apprenants aiment la poésie ou le théâtre. C'est ce que montrent, par exemple, les résultats d'une enquête menée en 2018 auprès des étudiants en licence qui apprennent le néerlandais à la KUL. Les résultats ont montré que 5\% parmi eux s'intéressent à la traduction de la poésie en L2, et $30 \%$ aux autres genres de textes littéraires. En revanche, la majorité préfère traduire des articles de journaux et de magazines (80\%) ainsi que des textes commerciaux (65\%). Toutefois, ce phénomène n'est pas lié au fait qu'il s'agit de langues L2, car les pourcentages de leur préférence pour la traduction de certains types de textes dans le cas des langues L1 présentent peu de différences (cf. poésie: 5\% ; textes littéraires: 40\%).

Dans les paragraphes ci-dessous, nous présentons la méthodologie et les résultats d'une étude de cas dans laquelle nous examinons dans quelle mesure des apprenants avancés de néerlandais langue étrangère utilisent des stratégies de médiation dans la traduction L2 d'un texte littéraire. Pour ce faire, les stratégies de traduction sont comparées dans la traduction (d'un 
fragment de texte) par un traducteur professionnel et locuteur natif (analyse 1 ), et par cinq étudiants en master de néerlandais (analyse 2).

\section{3. ÉTUDE DE CAS : TRADUCTION EN NÉERLANDAIS D'UN EXTRAIT DE LA PIÈCE CE THÉÂTRE PRZED SKLEPEM JUBILERA}

\subsection{OBJectif, HyPothÈse ET MÉTHOdologie}

L'objectif de cette étude de cas est d'analyser la mesure dans laquelle les apprenants avancés polonais du néerlandais langue étrangère de la KUL utilisent des stratégies de médiation dans la traduction d'un texte littéraire en L2. Ceci sera mis en rapport avec la mesure dans laquelle - à la recherche d'un transfer du sens plutôt que de la forme du texte source - ils auront recours à une traduction libre, et non pas à une traduction purement linguistique (cf. traduction mot à mot). Par conséquent, l'hypothèse de recherche est définie comme suit:

Les apprenants polonais avancés du néerlandais langue étrangère font peu appel aux stratégies de médiation lorsqu'ils traduisent un texte littéraire en $L 2$.

Cette affirmation repose sur nos années d'expérience dans l'enseignement de la traduction en L2 où nous avons pu témoigner d'un manque de sécurité linguistique de la plupart des apprenants qui - contrairement à un locuteur natif ont souvent recours à la traduction littérale qui consiste à traduire un texte mot à mot, c'est-à-dire sans aucun changement dans les mots ou dans leur ordre.

Pour le démontrer, nous analyserons la traduction néerlandaise d'un extrait de la pièce de théâtre Przed sklepem jubilera du Saint pape Jean-Paul II (dont nous présenterons la traduction néerlandaise dans la section 3.2.). Ce choix a été fait pour quatre raisons. Les deux premières sont liées à des facteurs externes et les deux suivantes à des facteurs textuels. La première raison liée aux facteurs externes est le fait qu'en 2018 notre université a fêté son $100^{\mathrm{e}}$ anniversaire, et que cette année (2020), elle célèbre le $100^{\mathrm{e}}$ anniversaire de la naissance du Saint qui fut professeur de la KUL. Deuxièmement, nous voulions profiter de cette occasion pour non seulement familiariser nos étudiants avec son œuvre littéraire dans sa traduction néerlandaise, mais aussi avec ses idées sur l'amour responsable dans le mariage. En ce qui concerne les facteurs textuels, nous avons opté pour (un passage de) cette 
pièce de Jean Paul II en raison de son langage simple, ce qui nous a amené à supposer que les apprenants feraient appel à une plus grande 'souplesse' au niveau de la traduction en L2. Néanmoins, vu la nature méditative du texte, l'apprenant sera obligé de faire certains choix afin de ne pas compromettre sa compréhension et sa clarté ${ }^{1}$. Enfin, nous voulions également examiner si la médiation dans la traduction peut également être pratiquée dans de textes littéraires qui ne sont pas entrecoupés de « realia » (définies comme « unités lexicales qui désignent une réalité particulière à telle ou telle culture $»^{2}$ ) qui sont souvent utilisés exclusivement dans le contexte de ce genre d'analyses.

Pour ce qui est de la méthodologie, nous avons effectué deux analyses (qui seront désignées par les termes 'analyse 1' et 'analyse 2') dans le but de mesurer et de comparer le degré de 'littéralité' de la traduction néerlandaise publiée en 1979 (cf. analyse 1 ci-dessous) et de celle de cinq apprenants polonais du néerlandais langue étrangère de la KUL - niveau B2/C1 (cf. analyse 2 ci-dessous). Ces traductions ont été faites par les étudiants inscrits en master de philologie néerlandaise dans le cadre des cours de traduction on L2 (polonais-néerlandais) pendant l'année 2018-2019.

Les deux analyses ont été basées sur le même fragment de texte, à savoir le texte source polonais de 30 lignes (KW-PL 20-31). En sélectionnant le fragment, nous avons tenu compte de la simplicité du langage (vocabulaire, structure des phrases). Comme déjà mentionné précédemment, la difficulté réside cependant dans la transmission du caractère méditatif du texte, comportant des phrases courtes avec des idées ici et là profondes formulées de manière compacte en langue étrangère ou L2.

Chaque analyse a été menée selon les critères classés par Chesterman (voir section 3.3.) qui prend en compte l'aspect grammatical, lexical et pragmatique de la traduction. Afin de déterminer quelles stratégies de traduction sont préférables dans les deux cas, nous avons effectué à chaque fois des recensements. Ensuite, le pourcentage a été calculé par type de stratégie (grammaticale, sémantique et pragmatique). Ces pourcentages sont basés sur les nombres totaux et le nombre de lignes du texte.

\footnotetext{
${ }^{1}$ À la chaire de néerlandais de l'Université catholique Jean-Paul II de Lublin (KUL), la traduction en L2 est enseignée dans le cadre des cours pratiques de néerlandais (L2). L'objectif de ce cours consiste principalement à promouvoir les compétences linguistiques des étudiants. Cette compétence comprend les sous-compétences suivantes: compétence lexicale, compétence grammaticale, compétence sémantique, compétence phonologique, compétence orthographique (CECR [2001] 87).

${ }^{2}$ Larousse, Dictionnaire français en ligne, https://www.larousse.fr/dictionnaires/francaismonolingue/, consulté le 05.2020.
} 
Dans les paragraphes suivants nous présentons quelques informations sur la traduction de la pièce de théâtre Przed sklepem jubilera (cf. 3.2.) en néerlandais, et dans le paragraphe 3.3. la taxonomie des stratégies de traduction définie par Andrew Chesterman qui est au cœur de notre analyse des stratégies de traduction (analyse 1 et analyse 2).

\subsection{PRZED SKLEPEM JUBILERA - DE WINKEL VAN DE JUWELIER}

La pièce Przed sklepem jubilera (fr. La boutique de l'orfèvre) est une pièce écrite par Karol Wojtyla (futur Jean Paul II) en 1960 alors qu'il était évêque de Cracovie. Elle porte le sous-titre: "Méditations sur le sacrement du mariage, se transformant de temps à autre en drame ». Przed sklepem jubilera a été publiée en quinze langues. Une centaine de millions d'exemplaires ont été vendus. En 1988, la pièce a été filmée avec Burt Lancaster dans l'un des rôles principaux. Frans van Dooren, le traducteur néerlandais de De winkel van de juwelier a basé son travail sur une édition italienne de $1979^{3}$. Au moment de sa publication, la pièce a été qualifiée par les journaux néerlandais de « leesdrama » ('drame de lecture') (Leewarder Courant) ou de « radiodrama » ('pièce radiophonique') (Trouw) parce qu'elle se compose non pas de dialogues, mais de prose et de poésie.

\subsection{Les stratégies traductionnelles de Andrew Chesterman}

Les trente stratégies distinguées par Andrew Chesterman sont réparties en trois catégories : les stratégies grammaticales, sémantiques et pragmatiques. Les catégories, qui se chevauchent partiellement, contiennent chacune dix stratégies principales (Chesterman 93). Nous avons choisi ce classement car il prend en compte l'aspect grammatical, lexical et pragmatique de la traduction. Nous les illustrerons par des exemples de la pièce: texte original (KW$\mathrm{PL}$ ) et traduction néerlandaise (KW-NL), sans passer par la traduction italienne. Comme les stratégies traductionnelles se chevauchent, une unité de traduction peut se retrouver dans plusieurs catégories. La seule catégorie qui ne peut être utilisée avec les autres est celle de la traduction littérale.

\footnotetext{
${ }^{3}$ Nous retrouvons dans l'article de Jezierska l'explication suivante : « La traduction italienne a été réalisée par Aleksandra Kurczab et Jerzy Pomianowski, un couple de Polonais qui a passé de nombreuses années en exil en Italie (ils ont émigré après la vague d'antisémitisme qui a commencé dans la Pologne communiste après les événements de mars 1968)».
} 


\section{STRATÉGIES GRAMMATICALES $(\mathrm{G})$}

Cette catégorie comporte les choix du traducteur en regard de la forme du texte (Chesterman 94-100).

\section{G1) Traduction littérale}

Une traduction aussi proche que possible de la forme de la source, tout en maintenant la grammaticalité de l'expression.

a. Andrzej wybrał mnie i poprosił o moją rękę. (KW-PL 1)

b. Andrek heeft mij gekozen en om mijn hand gevraagd. (KW-NL 13)

G2) Emprunts et calques

La traduction comporte des emprunts ou des néologismes formés à partir d'emprunts.

a. (Monika i Krzysztof) [KW-PL 28]

b. (Monica, Krzyszek) [KW-NL 57]

Dans le texte néerlandais nous voyons Krzyszek, la variante polonaise du prénom Krzysztof (fr. 'Christophe') sous la forme diminutive.

\section{G3) Transposition}

La traduction comporte des changements de parties du discours, par ex. nom $\rightarrow$ verbe, adjectif $\rightarrow$ adverbe, verbe $\rightarrow$ substantif

a. (...) musiałabym bowiem Patrzeć ponad głową Andrzeja. (KW-PL 1)

b. (...) omdat ik dan mijn blik Over Andreks hoofd heen had moeten laten gaan. (KW-NL13)

En polonais nous avons un verbe (cf. patrzeć) et en néerlandais un substantif (cf. blik - fr. 'regard').

\section{G4) Changement d'unité}

La traduction comporte des changements au niveau des unités constituantes, c'est-à-dire du morphème, du mot, du syntagme, de la proposition, de la phrase et du paragraphe.

a. Odpowiedziałam « tak »- nie od razu,

Ale po upływie kilka minut,

a jednak nie mogło być w ciągu tych minut żadnej refleksji, (...) (KW-PL 1) 
b. Ik antwoordde: 'Ja' - niet onmiddellijk maar na een paar minuten.

Toch gingen er in die minuten geen enkele gedachte door mij heen (...) (KW-NL 13)

Deux phrases au lieu d'une en néerlandais.

G5) Modification de la structure du syntagme

La traduction apporte des modifications soit au genre et au nombre etc. dans un syntagme nominal, soit à la personne, au temps ou au mode dans un syntagme verbal.

a. Nie było to pozwolenie nie zależne od aktu woli. (KW-PL 2)

b. Ik deed dat bewust, en niet zomaar op goed geluk. (KW-NL 14)

G6) Modification de la structure de la proposition

La traduction comporte des modifications portant par exemple sur l'ordre des éléments de la proposition, la diathèse (voix active et passive), la transitivité ou l'intransitivité etc.

a. (- w październiku są wczesne wieczory -) (KW-PL 6)

b. (in oktober is het vroeg donker) (KW-NL 20)

G7) Modification de la structure de la phrase

La traduction comporte des modifications du type de phrase, par ex. une proposition principale est rendue par une proposition subordonnée.

a. (...) mnie nagle błysnęła inna myśl: też o sygnałach - (KW-PL 4)

b. (...) flitste er een andere gedachte door mij heen, die ook met roepstemmen te maken had. (KW-NL18)

G8) Modification au niveau de la cohésion textuelle

La traduction comporte des modifications portant sur les références intratextuelles. Il s'agit d'ellipse, de substitution, de pronominalisation et de répétition.

a. Drzewa, drzewa - proste, szczuple pnie

tnq wysoko, wysoko od ócz, (..) (KW-PL 29)

b. Bomen, bomen, hoge slanke stammen

die het gezicht afhouden van de hemel, (...) (KW-NL12) 
G9) Modification du niveau de représentation

La traduction peut modifier le niveau de représentation d'éléments du texte. Par ex. au lieu du niveau syntaxique de l'original la traduction passe au niveau lexical comme dans l'exemple suivant:

a. Pamiętam zwłaszcza taki jeden miesiąc,

w tym miesiącu jeden taki wieczór - (KW-PL 3)

b. Ik herinner me nog heel goed, een maand, nee, een avond van die maand (...) (KW-NL 17)

Dans l'exemple ci-dessus, le néerlandais utilise une unité lexicale supplémentaire (cf. non dans non, un soir de ce mois) pour souligner qu'il s'agit d'un soir de ce mois. Dans le texte original polonais, cette signification (accent) est exprimée au niveau syntaxique, notamment par l'ordre des mots (cf. w tym miesiacu jeden wieczór).

G10) Modification de figure de style

La traduction comporte des modifications portant sur les tropes, par ex. le parallélisme, la répétition, l'allitération, le rythme.

a. (...) opowiedziała mi swe myśli, te, które snuły się w jej głowie (..) (KW-PL 5)

b. (...) biechtte zij (...) mij alles op wat haar die avond door het hoofd had gespeeld. (KW-NL 19)

a. Od kilka lat szla obok mnie (...) (KW-PL 5)

b. Zij trok al een paar jaar met mij op (KW-NL 20)

\section{STRATÉGIES SÉMANTIQUES (S)}

Cette catégorie comporte les choix du traducteur en regard de certains aspects de la signification de la phrase que nous avons trouvés dans Chesterman (102-107):

S1) Synonymie

La traduction comporte un synonyme, non pas un équivalent exact du mot présent dans le texte source, au but d'éviter par exemple la répétition.

a. (...) przez noc bieszczadzką szedł sygnal. (KW-PL 4)

b. (...) hun geroep doorkruiste het woud (...) (KW-NL 18) 
a. (...) a po chwili spytałam go jeszcze, czy wierzy w sygnały. (KW-PL 4)

b. (...) en een ogenblik later vroeg ik hem of hij in roepstemmen geloofde. (KWNL 19)

Dans l'exemple ci-dessous, le traducteur utilise par contre un synonyme, bien qu'il aurait pu se servir d'une traduction littérale (cf. plaat au lieu de ruit qui se traduit en polonais par okno) :

a. (...) po obu stronach wielkiej, przeźroczystej tafli (KW-PL 7)

b. (aan beide kanten van die grote doorschijnende ruit (KW-NL 20)

S2) Antonymie

La traduction comporte la négation d'un antonyme (ou vice-versa) de l'équivalent exact du mot présent dans le texte source.

a. (...) butów wygodnych na co dzień (...) (KW-PL 6)

b. (...) schoenen zonder pretenties (KW-NL 21]

a. (...) wzruszeniu bez słów. (KW-PL 8)

b. (...) een stille ontroering (KW-NL 23)

S3) Hyponymie

La traduction comporte des modifications du niveau hiérarchique des termes, en échangeant un hyponyme (mot au sens plus spécifique) avec l'hyperonyme (mot au sens plus général) ou vice-versa, ou encore un hyponyme avec un autre hyponyme (du même hyperonyme).

a. (...) wielkim, olbrzymim lustrem, (KW-PL 6)

b. (...) een enorme glazen wand, (KW-NL 21)

Dans la traduction néerlandaise le traducteur utilise glazen wand (fr. 'mur de verre' ou 'paroi en verre') au lieu d'une traduction littérale plus appropriée reusachtige spiegel (fr. 'grand mirroir').

S4) Inversion sémantique

La traduction se sert d'un terme au sens opposé à celui du texte source, par ex. vendre et acheter, avec d'autres modifications pour maintenir le sens de la phrase.

a. Na zewnątrz zmieniło się nic. (KW-PL 15)

b. Voor de buitenwereld verliep alles als vroeger. (KW-NL 36) 


\section{S5) Modification du niveau d'abstraction}

La traduction modifie le niveau d'abstraction d'éléments textuels.

a. (...) nie mogło też być żadnej walki motywów. (KW-PL 1)

b. (...) en er vond in mij geen enkele innerlijke strijd plaats (KW-NL 13)

S6) Modification de la distribution des sèmes

La traduction comporte des modifications de la distribution des sèmes dans les mots.

a. (...) szukajcie przystani miłości. (KW-PL12)

b. (...) moeten jullie (...) een ankerplaats vinden (...) om jullie liefde te beschutten. (KW-NL 30]

Dans l'exemple nous voyons une métaphore qui a été détaillée dans le texte néerlandais et qui fait référence au symbole de l'ancre (ankerplaats fr. 'l'ancrage') que les époux doivent trouver pour protéger leur amour conjugal. L'ancre évite au bateau d'être poussé par le vent ou le courant sur les rochers. Elle doit reposer sur un fond solide qui est le lieu saint, le lieu d'accès à Dieu (cf. Hébreux 6.19: „Cette espérance, nous la possédons comme une ancre de l'âme, sûre et solide ; elle pénètre au-delà du voile".)

a. Powinien też już zamknąć I wrócić do domu. (KW-PL 14)

b. In plaats van tot laat in de avond door te werken, is het beter om te sluiten en naar huis te gaan. (KW-NL 32)

S7) Modification du degré d'intensité

La traduction comporte des modifications du degré d'intensité ou de la mise en relief.

a. Cóż za dziwne rzemiosło. (KW-PL 8)

b. Wat een uitzonderlijke kunst ! (KW-NL 23)

Dans la traduction néerlandaise le traducteur a également ajouté un point d'exclamation.

S8) Paraphrase

La traduction est plus libre, ne comportant pas tous les sèmes de la phrase source. C'est la ressemblance pragmatique qui est mise en relief. 
a. (...) pójdziemy potem aleją, kilkadziesiąt metrów, kilkaset (...) (KW-PL 11)

b. (...) we zullen een eindje met jullie meelopen, een flink stuk (...) (KW-NL 29)

S9) Modification de trope

Cette stratégie est utilisée pour la traduction de tropes rhétoriques, c'està-dire les figures de style, de façon similaire à la stratégie grammaticale portant sur la traduction des figures de style.

a. (...) butów wygodnych na co dzień (...) (KW-PL 6)

b. (...) schoenen zonder pretenties (KW-NL 21)

Dans le texte néerlandais, nous voyons apparaître une personnification ('des chaussures sans prétention') qui n'est pas présente dans le texte original en polonais.

\section{S10) Autres modifications sémantiques}

Ces changements peuvent inclure par exemple des modifications au niveau de la traduction des sens physiologiques, comme la vision, le toucher ou l'ouïe.

a. To sklep jubilera. (KW-PL 8)

b. Kijk naar de winkel van de juwelier. (KW-NL 23)

Dans le texte original polonais il n'y pas de mots qui réfèrent à la traduction du mot kijk (fr. 'regardes' du verbe 'regarder'). En d'autres mots la perception visuelle est ici exprimée par le verbe.

\section{STRATÉGIES PRAGMATIQUES (P)}

Cette catégorie comporte les choix du traducteur en regard de la sélection d'informations dans la langue cible. Dans ce choix, le traducteur se laisse guider par ce qu'il sait des futurs lecteurs de la traduction. Des stratégies pragmatiques influencent le message lui-même (Chesterman 108-112).

\section{P1) Filtrage culturel}

Ce filtrage concerne l'adaptation de la terminologie de la langue source à celle de la langue cible. Cette stratégie est également appelée naturalisation, domestication ou adaptation (Chesterman 108).

a. (...) noc bieszczadzką (KW-PL 4)

b. de nacht van het gebergte (KW-NL 19) 
Le manque de traduction de l'adjectif bieszczadzki provient du fait qu'il n'a pas été saisi dans la traduction italienne, pourtant il a une charge culturelle. Cet adjectif est dérivé du nom des montagnes polonaises Bieszczady qui évoquent la beauté, la pureté, l'étendue, la liberté.

P2) Modification d'explicitation

La traduction comporte des modifications au niveau de l'implicite ou de l'explicite dans le texte.

a. I wiem, że nie mogę iść dalej. (KW-PL 5)

b. En ik weet het, ik kan niet meer alleen lopen. (KW-NL 20)

P3) Ajout ou omission d'information

Soit la traduction comporte de l'information qui n'est pas présente dans le texte original :

a. Andrzej jest wyższy ode mnie na tyle, że muszę sobie trochę dodać wzrostu (KW-PL 6)

b. Andrek is groter dan ik, zoveel groter dat er voor mij meer dan genoeg reden is om groter te willen worden (KWNL 21)

soit une partie de l'information du texte original est omise :

a. Stefan słuchał, ale nie odczułam, aby przejął się tym, co mówiłam. (KW-PL 14)

b. Stefan luisterde naar mij, maar zonder zich druk te maken over datgene wat ik zei. (KW-NL 35]

P4) Modification interpersonnelle

Le style général de la traduction et la relation entre le texte et le lecteur sont modifiés, engendrant par ex. des changements du niveau de formalité ou du vocabulaire technique.

a. ... parę taktów lekkiej muzyki dobrze dziala. (KW-PL 22)

b. Wat muziek zal ons goed doen. (KW-NL 46)

En polonais, nous avons une construction impersonnelle, tandis qu'en néerlandais un pronom personnel ons (fr. 'nous'). 


\section{P5) Modification illocutionnaire}

La traduction comporte des changements au niveau des actes de langage. Par exemple, le remplacement du mode indicatif par le mode impératif déclenche la modification illocutionnaire d'une affirmation en une demande.

a. Nie czujesz zdroju, a płomień cię trawi. Prawda? (KW-PL 25)

b. Je merkt de bron niet op, maar het vuur verslindt je. (KW-NL 51)

En polonais il y a une question, une demande d'affirmation, tandis que dans la traduction néerlandaise nous avons une affirmation.

P6) Modification au niveau de la cohérence textuelle

La traduction comporte des modifications au niveau de l'ordre logique de l'information présentée dans le texte. Par exemple, deux paragraphes du texte source peuvent être combinés en un seul dans le texte cible.

a. Adam powiedział znienacka,gdyśmy po chwili stanęli na tym samym miejscu: (...) [KW-PL19]

b. Toen we opnieuw voorbij de winkel kwamen, zei Adam onverwachts: (...) [KW-NL 42]

P7) Traduction partielle

La traduction n'est par exemple qu'un résumé ou une transcription des phonèmes du texte original.

a. Zmrok zapada (...) [KW-PL 28]

b. Schemering. [KW-NL 55]

P8) Modification de visibilité

La traduction comporte des modifications au niveau de la visibilité de l'auteur ou du traducteur, par ex. par des notes de bas de page expliquant des jeux de mots. Nous n'avons pas trouvé d'illustrations dans le texte.

\section{P9) Réécriture}

Si le texte original est mal écrit ou fautif, le traducteur n'a d'autre option que de faire de grandes modifications, c'est-à-dire avoir recours à la réécriture. Dans notre analyse nous n'avons pas trouvé d'exemples de réécriture. 
P10) Autres modifications pragmatiques

Ces modifications peuvent porter par exemple sur la mise en page ou dans le choix du dialecte de la langue cible. Dans notre analyse nous n'avons pas trouvé d'exemples d'autres modifications pragmatiques.

$\mathrm{Vu}$ que les différences observées ci-dessus ne portent pas directement sur le rapport entre le texte polonais et la traduction en néerlandais car l'étape intermédiaire (la traduction néerlandaise à partir du texte italien) n'était pour nous pas accessible, il serait intéressant de faire une analyse des stratégies à partir de la traduction en italien par rapport au texte original en polonais. Il serait souhaitable par la suite de revoir la traduction néerlandaise, et de la vérifier par rapport au texte original.

\section{ETUDE DE CAS : ANALYSE 1 ET ANALYSE 2}

\subsection{INTRODUCTION: REMARQUES GÉNÉRALES SUR LA TRADUCTION DE 1979}

Avant d'entamer l'analyse de la traduction d'un fragment sélectionné de Przed sklepem jubilera, nous aimerions - en guise d'introduction - présenter quelques remarques générales sur l'ensemble de la traduction néerlandaise. En lisant le texte en entier, nous avons l'impression qu'il s'agit d'une traduction exotisante où le traducteur a préservé la spécificité de la culture source (Grit 43-44). Dans les sous-titres utilisés dans la pièce le traducteur utilise les mêmes prénoms qui figurent dans la traduction italienne (Jezierska 75). De ce fait - tout comme dans la traduction italienne - la traduction néerlandaise contient d'une part des diminutifs de certains prénoms polonais qui n'ont pas été modifiés comme par exemple Kryszek (KW-NL 57) (Jezierska 75). D'autre part on y retrouve des prénoms dont la forme a été adaptée à l'orthographe italien (Jezierska 75), comme par exemple dans le cas du prénom Monica (KW-NL 57) qui en polonais s'écrit avec un $k$. D'autre part on retrouve dans la traduction néerlandaise des diminutifs qui ne figurent pas dans la traduction italienne et n'existent ni en polonais, ni en néerlandais comme par exemple Andrek (cf. it. Andrea; Jezierska 75) (KW-NL 13) (cf. en polonais: Jędrek ou Jędruś) et Maroziszek (KW-NL 37).

Ce qui par contre remet en question qu'il s'agit d'une traduction exotique, c'est par exemple la traduction du terme (géographique) culturel polonais noc bieszczadzka (KW-PL 4) ou l'adjectif bieszczadzki (dérivé des mon- 
tagnes Bieszczady) a été omis (cf. pl. noc bieszczadzka; nl. de nacht van het gebergte).

Une autre remarque concerne la traduction du titre en néerlandais dans lequel la préposition przed (nl. voor) a été omise. En effet, le traducteur travaillait à partir de la traduction italienne du texte où la préposition est également absente dans le titre - La bottega dell'orefice. Néanmoins, la présence de la préposition przed nous semble importante, car les personnages se tiennent souvent 'en méditation' devant la vitrine d'un orfèvre et y prennent des décisions importantes:

Stoimy wciąż przed tym sklepem wybierając wspólnie nasz los. (KW-PL 9)

En we staan nog steeds voor de winkel terwijl we

ingespannen proberen ons gemeenschappelijk levenslot

te kiezen. (KW-NL 25)

Lors d'une lecture et d'une comparaison plus approfondies des deux textes, nous avons également aperçu un nombre important de stratégies sémantiques, notamment des traductions libres. Un exemple de traduction libre (= S8) au niveau de la phrase est le passage suivant :

- Jak blisko przeszła wówczas obok mnie! (KW-PL 5)

- Hoe liet zij die nacht voor mij tot leven komen! (KW-NL 19) (fr. 'Combien a t'elle pour moi donné vie à la nuit!')

Nous retrouvons aussi des traductions libres au niveau des mots. Un exemple est la traduction du mot sygnaly (KW-PL 1) par roepstemmen (KWNL 11) (fr. 'voix d'appel') qui réfère aux cris que les personnages entendent un soir dans les montagnes. Toutefois, en poursuivant la lecture, nous pouvons nous demander si le mot sygnaty n'aurait pas pu être mieux traduit en néerlandais par signalen (fr. 'signal'). Dans un autre passage, l'auteur fait notamment référence aux signes d'avertissement ou aux événements de la vie qui peuvent inciter une personne à faire certains choix. Ces signaux font partie d'une "carte compliquée" (cf. skomplikowanej mapie) qui ne peut être interprétée ou comprise qu'au fil du temps :

Po kilku latach widzę to wyraźnie, że drogi, które powinny się rozbiec, zbliżyły nas właśnie do siebie.

Tych kilka lat to czas bezcenny, by zorientować się w skomplikowanej

mapie sygnałów i znaków. (KW-PL 5) 
$\mathrm{Nu}$ de jaren voorbij zijn, zie ik duidelijk

hoe onze wegen uit elkaar hadden kunnen gaan, terwijl ze ons juist tot elkaar gebracht hebben.

Deze paar jaren zijn onbetaalbaar geweest,

ze hebben ons in de gelegenheid gesteld om ons te

oriënteren op de ingewikkelde

kaart van roepstemmen en signalen. (KW-NL 20)

La traduction en néerlandais kaart van signalen en tekenen (fr. 'carte de signaux et de signes') nous semble plus appropriée dans ce contexte car - en outre - le mot teken est la traduction littérale du mot znak.

Un exemple d'une traduction libre accompagnée d'une description (cf. la traduction du mot jeziorek par bekkens met stilstaand water [fr. 'des bassins d'eau stagnante'] au lieu de meer [fr. 'lac']) :

- Zwłaszcza owego wieczoru, gdy przy zejściu zastała noc.

Nie zapomnę nigdy tych jeziorek, co zaskoczyly nas po drodze

jak gdyby dwie cysterny niezgłębionego snu. (KW-PL 3-4)

- Vooral die avond toen we tijdens de afdaling door de duisternis werden verrast.

Ik zal nooit de bekkens met stilstaand water vergeten

waarbij we toevallig terechtkwamen:

twee putten vol bodemloze slaap. (KW-NL 17-18)

Ici nous pouvons également nous demander si le traducteur n'aurait pas pu se servir d'une traduction littérale en traduisant cysterny par cisterne (fr. 'cisterne') au lieu de put (fr. 'puit'). En utilisant le mot put (fr. 'trou', 'puit') l'image de la paix profonde évoquée par la profondeur d'une citerne (associée au profond sommeil) rendu par la métaphore en polonais semble disparaître dans la traduction. Vu qu'en néerlandais le terme cisterne possède une connotation plutôt technique, le terme put semble néanmoins plus approprié.

Une stratégie pragmatique que nous observons sur toute la ligne de traduction - et qui peut être comptabilisée sous P10 (autres changements pragmatiques) - est l'altération systématique de la mise en page du texte en néerlandais: lorsque en polonais une phrase se termine, une nouvelle phrase apparaît souvent dans le texte néerlandais, et vice versa (cf. exemple cidessous). Ici et là, différents signes de ponctuation sont utilisés dans la traduction, ou des morceaux de texte sont placés entre parenthèses rondes dans le texte polonais. En outre nous apercevons à plusieurs reprises l'élimination d'espaces blanches qui marquent le début d'un nouveau paragraphe: 
[espace blanche]

Tak trzeba.

dziś widzę, że mój kraj jest także jej krajem,

a przecież marzyłem, by przerzucić pomost - (KW-PL 5)

(...)

kaart van roepstemmen en signalen

Zo moest het gebeuren.

$\mathrm{Nu}$ zie ik dat míjn wereld ook de hare is.

En toch droomde ik er alleen maar van om een brug te slaan. (KW-NL 20)

Dans cet exemple nous apercevons également une stratégie sémantique, à savoir une S5 (changement de niveau d'abstraction) car kraj (fr. 'pays') est traduit par un mot ayant un sens plus général, notamment monde, même si dans le texte polonais l'auteur utilise des mots liés au voyage tels qu'une route, une carte (du pays) et la découverte éventuelle que le voyage conduit à quelqu'un qui se trouve dans le même pays, de sorte que le rêve de construire des ponts s'avère en fin de compte inutile.

Finalement, nous avons également trouvé une erreur dans la traduction d'une passage, notamment dans la phrase suivante:

a. Nikt mu nie każe powracać. (KW- PL 27)

b. Niemand zegt haar dat ze terug moet keren. (KW-NL 54)

Dans le texte polonais $m u$ est le pronom personnel dans le cas de datif, et il réfère à Stefan ; traduction littérale française: 'Personne ne lui ordonne de rentrer'. Par contre, dans le texte néerlandais (fr. 'Personne ne lui dit de rentrer'), le pronom lui - également dans le datif - est au féminin et réfère à Anna.

\subsection{Resultats de L'Analyse 1 - Traduction de 1979}

\section{STRATÉGIES GRAMMATICALES}

En comparant les textes, nous avons découvert trois stratégies grammaticales. A la ligne 11 du fragment, nous remarquons une stratégie G3 (transposition), car Andrzej a été remplacé par hij (fr. 'lui') (cf. un nom propre est remplacé par un pronom) :

- (...) o Andrzeju i o sobie samej (KW-PL 6)

- (...) aan hem en aan mezelf (KW-NL 21] 
En outre, nous apercevons dans la traduction néerlandaise à la ligne 25-26 (cf. Annexe) du fragment analysé (KW-NL 22) une stratégie G9 (modification du niveau de représentation), car dans le texte polonais la phrase avec le noyau de etalage fait partie d'un adverbe (complément circonstanciel) et dans la traduction néerlandaise d'un complément d'objet direct:

- Potem jeszcze patrzyliśmy wspólnie na witrynę sklepu jubilera. (KW-PL 7)

- Daarna bekeken we samen lange tijd de etalage van de juwelier. (KW-NL 22)

Dans ce fragment, il est également question de la stratégie G5 (modification de la structure du syntagme), cf. «witryna sklepu jubilera » par rapport à « de etalage van de juwelier».

Tableau 1: Nombre de stratégies $G$ dans la traduction néerlandaise (analyse 1)

\begin{tabular}{|c|c|c|}
\hline G3 & G5 & G9 \\
\hline 1 & 1 & 1 \\
\hline
\end{tabular}

\section{STRATÉGIES SÉMANTIQUES}

L'analyse 1 nous montre que le fragment contient 20 stratégies sémantiques: douze stratégies S8 (paraphrase), trois stratégies S7 (modification du degré d'intensité), deux stratégies S2 (antonymie), une stratégie S5 (changement de niveau d'abstraction), une stratégie S9 (modification de trope) en une stratégie S1 (traduction littérale).

Tableau 2: Nombre de stratégies S dans la traduction néerlandaise (analyse 1)

\begin{tabular}{|c|c|c|c|c|c|}
\hline S1 & S2 & S5 & S7 & S8 & S9 \\
\hline 1 & 1 & 1 & 3 & 12 & 1 \\
\hline
\end{tabular}

Exemple d'une stratégie S8:

- Szukałam wzrokiem butów z wysokim obcasem. (KW-PL 6)

- maar ik zocht nu net schoenen met hakken (KW-NL 21) 
Exemple d'une stratégie S7:

- Ja mówiłam mu o tym krawacie, (KW-PL 7)

- Ik had het over de stropdas (KW-NL 22]

Exemple d'une stratégie S2:

- a więc jednak myślałam o Andrzeju, (KW-PL 6)

- Het is dus niet waar dat ik niet aan Andrek dacht, (KW-NL 21)

Exemple d'une stratégie S5:

- Szukałam wzrokiem butów z wysokim obcasem. (KW-PL 6)

- Ik was op zoek naar schoenen met hoge hakken. (KW-NL 21)

Exemple d'une stratégie S9:

- (...) wiele butów wygodnych na co dzień, (KW-PL 6)

- (...) allerleid schoenen zonder pretenties, (KW-NL 21)

\section{STRATÉGIES PRAGMATIQUES}

En ce qui concerne les stratégies pragmatiques, nous avons trouvé dix stratégies P, en particulier quatre stratégies P2 (= modification d'explicitation), quatre stratégies P3 (= ajout ou omission d'information) et deux stratégies $\mathrm{P} 10$-strategieën (= autres modificatons pragmatiques):

Tableau 3: Nombre de stratégies $P$ dans la traduction néerlandaise (analyse 1)

\begin{tabular}{|c|c|c|}
\hline P2 & P3 & P10 \\
\hline 4 & 4 & 2 \\
\hline
\end{tabular}

Exemples d'une stratégie P2:

- Andrzej jest wyższy ode mnie na tyle (...) (KW-PL 6)

- Andrek is groter dan ik, zoveel groter (...) (KW-NL 21)

- (...) wybierzemy dla siebie obrączki". (KW-PL 7)

- (...) we moeten onze trouwringen uitzoeken.' (KW-NL 22) 
Exemples de stratégies P3:

- Potem jeszcze patrzyliśmy wspólnie (KW-PL 7)

- Daarna keken we samen lange tijd (KW-NL 22)

- Andrzej jest wyższy ode mnie na tyle, że muszę sobie trochę dodać wzrostu (KW-PL 6)

- Andrek is groter dan ik, zoveel groter dat er voor mij meer dan genoeg reden is om groter te willen worden (KW-NL 21)

Dans les cas où il est question de stratégies P10 (= autres modifications pragmatiques), il s'agit avant tout de modifications de la mise en page (cf. disposition des phrases et des paragraphes). Dans le fragment sélectionné pour l'analyse 1, nous avons trouvé deux exemples de ce type de stratégie.

Exemple d'une stratégie P10:

- Potem jeszcze patrzyliśmy wspólnie na witrynę sklepu jubilera, gdzie w pudełeczkach powleczonych wewnątrz aksamitem spoczywały różne precjoza.

- Daarna keken we allebei Aan [sic] de etalage van de juwelier, waar in doosjes met een fuwelen binnenkant verschillende sieraden lagen.[RK]

Dans cet exemple nous voyons une modification au niveau du nombre de phrases.

Le tableau 4 ci-dessous montre la répartition en pourcentage des trois stratégies que nous avons trouvées dans le fragment de texte sélectionné (30 lignes) :

Tableau 4: Pourcentage de stratégies dans la traduction néerlandaise (analyse 1)

\begin{tabular}{|c|c|c|}
\hline Stratégies G & Stratégies S & Stratégies $\mathbf{P}$ \\
\hline $10 \%$ & $66,6 \%$ & $23,3 \%$ \\
\hline
\end{tabular}

Les résultats de l'analyse 1 nous montrent que la traduction contient principalement des stratégies $\mathrm{S}$. En ce qui concerne le type de stratégie $\mathrm{S}$, nous avons aperçu principalement la stratégie S8 ou la paraphrase. La fréquence 
élevée de ce type de stratégie s'explique par le fait que cette pièce contient peu de dialogues. Il s'agit principalement de la prose qui représente les pensées des personnages, et dans laquelle chaque phrase est étalée sur au moins deux lignes (de six à huit mots au maximum).

Les stratégies $\mathrm{P}$ occupent la deuxième place. En ce qui concerne ces stratégies, nous avons trouvé en particulier la stratégie P2 et la stratégie P3. Il s'agit de stratégies dans lesquelles d'une part des informations sont ajoutées ou omises et, d'autre part, certaines informations sont rendues plus implicites ou explicites. À cet égard, nous avons constaté que certains ajouts ou omissions et/ou modifications d'explicitation semblent inutiles (cf. exemples des stratégies $\mathrm{P} 2$ et $\mathrm{P} 3$ au paragraphe 5.2.3.). Comme le transfer de ces phrases en L2 n'implique pas un réel problème de traduction polono-néerlandais, nous soupçonnons qu'ils ont été copiés/traduits à partir du texte italien.

Enfin, lorsque nous examinons la fréquence des stratégies grammaticales, nous constatons que leur nombre dans le fragment de texte étudié n'en contient que $10 \%$. Cela est dû au fait que la traduction contient de nombreuses paraphrases, ce qui signifie qu'une analyse des changements au niveau syntaxique n'est pas incluse.

\subsection{AnAlyse 2 - traductions faites Par Des Étudiants Polonais}

Dans ce paragraphe, nous présentons les résultats de l'analyse 2 dans laquelle nous examinons quelles stratégies sont le plus fréquemment utilisées dans la traduction néerlandaise du même fragment par cinq étudiants polonais. Dans cette analyse il s'agit des traductions directes du texte original polonais vers le texte néerlandais. Comme nous l'avons déjà souligné, nous sommes partis de l'hypothèse suivante:

Dans la traduction (L2) literaire les étudiants utilisent le plus souvent la stratégie de traduction littérale.

Nous n'avions pas l'intention de nous concentrer sur les erreurs linguistiques, mais sur les 'mécanismes' ou stratégies utilisés par les étudiants pour transmettre le contenu/message au lecteur. Nous avons supposé que lors de la traduction de textes en L2, les apprenants utilisent des stratégies les moins invasives, en particulier la traduction littérale (G1) ou une traduction mot à mot, ce qui est lié à l'insécurité linguistique.

Dans l'analyse 2 nous avons remarqué que la plupart des ont maintenu les prénoms polonais Andrzej et Teresa. Par contre, certains ont utilisé le pré- 
nom Andreas au lieu de Andrzej, et Trees (forme utilisée en langue parlée en néerlandais) ou Therese (écrit sans accents par les étudiants. En néerlandais ce prenom figure avec ou sans accents: Thérèse ou Therese) au lieu de Tere$s a$. Il convient également de noter que tous les élèves ont traduit le mot polonais sygnaty par le mot néerlandais seinen qui aurait pu être un choix plus approprié que roepstem (fr. 'voix d'appel') vu que dans un autre passage, l'auteur utilise également le terme sygnaty pour faire référence aux signes d'avertissement ou aux événements de la vie qui peuvent inciter une personne à faire certains choix (cf. [KW-PL 5]).

\section{STRATÉGIES GRAMMATICALES}

L'analyse des stratégies grammaticales a montré que ce type de stratégie représentait la plus grande proportion avec un nombre total de 102 pour cinq traductions. En ce qui concerne la stratégie de type G, 90 stratégies G1 (= traductions littérales) ont été appliquées. Cela signifie que la fréquence moyenne des stratégies G1 par traduction est de $68 \%$ et que plus de la moitié du texte a été traduit littéralement.

Lors de l'analyse des traductions des etudiants (= analyse 2 ), nous avons également trouvé six fois la stratégie G6 (modification de la structure de la proposition), deux fois la stratégie G5 (modification de la structure du syntagme) et deux fois la stratégie G3 (transposition). Exprimé en pourcentage, cela nous donne le résultat suivant :

Tableau 5: Le nombre et le pourcentage des stratégies $\mathrm{G}$ dans les traductions $L 2$ des étudiants (analyse 2 )

\begin{tabular}{|c|c|c|c|}
\hline $\mathbf{G 1}$ & $\mathbf{G 3}$ & $\mathbf{G 5}$ & $\mathbf{G 6}$ \\
\hline 90 & 2 & 2 & 6 \\
\hline $\mathbf{6 0 \%}$ & $\mathbf{1 , 3} \%$ & $\mathbf{1 , 3} \%$ & $\mathbf{4 \%}$ \\
\hline
\end{tabular}

Exemple de la stratégie G1:

Szukałam wzrokiem butów z wysokim obcasem.

Ik zocht met mijn blik naar schoenen met hoge hakken. (SL) ${ }^{4}$

\footnotetext{
${ }^{4}$ Dans l'analyse 2 les abréviations utilisées auprès des exemples font référence aux initiales de l'étudiant.
} 


\section{STRATÉGIES SÉMANTIQUES}

En ce qui concerne les stratégies sémantiques, nous avons trouvé un total de 35 stratégies: 32 stratégies S8, deux stratégies S2 et une stratégie S1:

Tableau 6: Le nombre et le pourcentage des stratégies $\mathrm{S}$ dans les traductions $L 2$ des étudiants (analyse 2 )

\begin{tabular}{|c|c|c|}
\hline S1 & S2 & S8 \\
\hline 1 & 2 & 32 \\
\hline $0,6 \%$ & $1,3 \%$ & $21,3 \%$ \\
\hline
\end{tabular}

Exemples de stratégies S8

Andrzej jest wyższy ode mnie na tyle, że muszę sobie dodać trochę wzrostu (KW-PL 6)

Andrzej is zoveel hoger dan ik

Daarom moet ik een beetje hoger zijn. (SL)

(...) lecz najbardziej wodziłam wzrokiem za butami na wysokim obcasie. (KW-PL 6)

maar ik zocht vooral

naar hoge hakken. (RK)

\section{STRATÉGIES PRAGMATIQUES}

Dans les traductions des étudiants, nous avons découvert un total de treize stratégies P: cinq stratégies P10 (= autres modifications pragmatiques), quatre stratégies P3 (= ajout ou omission d'information) et quatre stratégies P2 (= modification d'explicitation). La stratégie P10 indique les changements dans la mise en page, dans la répartition d'une phrase sur plus ou moins de lignes que dans le texte source (cf. exemple au paragraphe 5.1 Stratégies pragmatiques).

Tableau 7: Le nombre et le pourcentage des stratégies $P$ dans les traductions L2 des étudiants (analyse 2)

\begin{tabular}{|c|c|c|}
\hline P2 & P3 & P10 \\
\hline 4 & 4 & 5 \\
\hline $2,6 \%$ & $2,6 \%$ & $3,3 \%$ \\
\hline
\end{tabular}


Exemple d'une combinaison d'un stratégie P2 et P3:

- a więc jednak myślałam o Andrzeju,

o Andrzeju i o sobie samej

- dus ik dacht aan Andrzej, aan ons beiden (RK)

Dans l'exemple ci-dessus, la mot jednak dans la première phrase a été omis dans la traduction néerlandaise (=P3 ou ommission d'information). Dans la deuxième phrase par contre, la traduction neerlandaise beiden modifie l'explicitation du texte orginal polonais (cf. o Andrzeju et o sobie samej) $(=\mathrm{P} 2)$.

Le tableau 4 ci-dessous montre la répartition en pourcentage des trois stratégies que nous avons trouvées dans le fragment de texte sélectionné (30 lignes) :

Tableau 8: Pourcentage de stratégies

dans les traduction L2 des étudiants (analyse 2)

\begin{tabular}{|c|c|c|}
\hline Stratégies G & Stratégies S & Stratégies $\mathbf{P}$ \\
\hline $68 \%$ & $23,33 \%$ & $8,6 \%$ \\
\hline
\end{tabular}

Lorsque nous comparons les pourcentages des trois stratégies de l'analyse 1 et 2 (cf. le tableau 4 et 8 ), nous voyons que le traducteur professionnel applique surtout des stratégies sémantiques $(66,6 \%)$ et pragmatiques $(23,3 \%)$. En ce qui concerne les stratégies sémantiques, il utilise principalement la paraphrase (= stratégie S8). Quant aux stratégies pragmatiques, il fait surtout appel à la stratégie P2 et la stratégie P3. Ici il s'agit de stratégies dans lesquelles d'une part des informations sont ajoutées ou omises, et d'autre part certaines informations sont rendues plus implicites ou explicites. Ici nous devons tenir compte du fait que la langue du texte source de la traduction de 1979 est l'italien. Il se peut que sous l'influence de l'italien certaines phrases soient plus détaillées et plus descriptives dans la traduction néerlandaise de 1979 que le texte original polonais. Cela peut encourager une révision de la traduction néerlandaise.

D'autre part, nous constatons que les étudiants font surtout appel a des stratégies grammaticales (cf. 68\%) et sémantiques (cf. 23,33\%). Au niveau des stratégies grammaticales, ils ont surtout recours à une traduction littérale (cf. G1=60\%). Pour ce qui est les stratégies sémantiques, nous voyons que la paraphrase (stratégie S8) occupe la première place (cf. 21,3\%). Vu que 
l'utilisation de la stratégie S8 ou la paraphrase prouvent que le traducteur traduit plutôt le sens que le texte (cf. mot à mot), les résultats de cette étude nous montrent qu'en traduisant un texte littéraire en L2, les apprenants appliquent effectivement des stratégies de médiation.

\section{CONCLUSION}

Sur la base des données ci-dessus des deux analyses (analyse 1 et 2), nous pouvons conclure que les apprenants avancés du néerlandais utilisent principalement des stratégies grammaticales dans la traduction d'un texte littéraire en L2. Lorsque nous mettons ce résultat en relation avec l'hypothèse de recherche, nous constatons que sa validité a été confirmée. Ainsi, les apprenants avancés utilisent principalement des traductions littérales en L2. Néanmoins, le pourcentage de stratégies $\mathrm{S}$, en particulier la S8, montre que, dans une certaine mesure, les étudiants utilisent également la paraphrase afin de ne pas compromettre la clarté et la compréhension du texte (lorsqu'une traduction littérale le provoquerait). C'est un signal qui va dans le sens d'un certain 'sens de la médiation'. Cela nous amène à la conclusion générale que la traduction L2 d'un texte littéraire - comme, par exemple, une pièce de théâtre - peut effectivement servir d'activité de médiation pour former les compétences de communication interculturelle des apprenants en langues étrangères. Bien entendu, cette étude de cas doit encore être réalisée à une plus grande échelle afin de confirmer ces résultats de manière quantitative.

\section{BIBLIOGRAPHIE}

Carreres, Angeles. « Strange Bedfellows : Translation and Language Teaching». Paper delivered at the Sixth Symposium on Translation, Terminology and Interpretation in Cuba and Canada, 2006, pp. 1-21. CTTIC - Canadian Translators, Terminologists and Interpreters Council, cttic.org/ACTI/2006/papers/Carreres.pdf. Consulté 04.2020.

Carreres, Angeles. «Translation as a Means and as an End: Reassessing the Divide ». The Interpreter and Translator Trainer, vol. 8, issue 1, 2014 : Translation in the Language Classroom: Theory, Research and Practice, pp. 123-135.

CECR [2001] : Conseil de l'Europe. Le Cadre européen commun de référence pour les langues Apprendre, Enseigner, Évaluer. Unité des Politiques linguistiques, 2001.

CECR [2018] : Conseil de l'Europe. Le Cadre européen commun de référence pour les langues Apprendre, Enseigner, Évaluer. Volume complémentaire avec de nouveaux descriptifs. Unité des Politiques linguistiques, 2018. 
Chesterman, Andrew. Memes of Translation : The Spread of Ideas in Translation Theory, $2^{\mathrm{e}}$ édition. Benjamins Translation Library, 2000 ( $1^{\mathrm{e}}$ éd. 1997).

Cook, Guy. Translation in Language Teaching. Oxford University Press, 2010.

Council of Europe. The Common European Framework of Reference for Languages: Learning, Teaching, Assessment. Language Policy Unit, 2001.

Duff, Alan. Translation. Oxford University Press, 1989.

Grit, Diederik. « De vertaling van Realia ». Filter, vol. 4, 1997, pp. 42-48.

Howatt, Anthony Philip Reid. A History of English Language Teaching. Oxford University Press, 1984.

Jezierska, Beata. «La valeur sémantique ajoutée et perdue dans la traduction en italien du drame polonais Przed sklepem jubilera de Karol Wojtyła », dans ce volume, pp. 53-68.

Larousse, Dictionnaire français en ligne, www.larousse.fr/dictionnaires/francais-mono lingue/. Consulté le 05.2020.

Laviosa, Sara. Translation and Language Education. Routledge, 2014.

Missinne, Lut. « Vertalen als hulpmiddel bij het literatuuronderwijs ». Colloquium Neerlandicum (2000). Perspectieven voor de internationale neerlandistiek in de 21ste eeuw. Handelingen Veertiende Colloquium Neerlandicum. Internationale Vereniging voor Neerlandistiek, 2001, pp. 411-418.

\section{ARTICLES DE JOURNAUX}

Leeuwarder courant: hoofdblad van Friesland. « KRO zendt hoorspel van paus uit. Leeuwarden: Stichting Leeuwarder courant». 22-05-1979. Delpher. www.delpher.nl/nl/kranten/ view? query=winkel+juwelier+wojtyla\&coll=ddd\&identifier $=$ ddd:010621105:mpeg21:a0161\&r esultsidentifier=ddd:010621105:mpeg21:a0161. Consulté le 04.2020.

Trouw. «KRO zendt hoorspel van paus uit. Meppel : Organisatie trouw ». 02-06-1979. Delpher. www.delpher.nl/nl/kranten/view?query=winkel+juwelier+wojtyla\&coll=ddd\&identifier= ABCDDD:010824274:mpeg21: a0408\&resultsidentifier=ABCDDD:010824274: $\operatorname{mpeg} 21$ : a0408. Consulté le 04.2020 .

\section{TEXTES SOURCES}

KW-NL : Wojtyła Karol - Jawień Andrzej. De winkel van de juwelier. Vertaald en ingeleid door Frans van Dooren. Kwadraat, 1996/1979.

KW-PL : Wojtyła, Karol. Przed sklepem jubilera. Medytacja o sakramencie matżeństwa przechodzaca chwilami w dramat. Wydawnictwo TUM, 1960. (Przedruk z: Karol Wojtyła. Poezje i dramaty. Wydawnictwo Znak, 1980). 


\title{
LA TRADUCTION LITTÉRAIRE COMME OUTIL DIDACTIQUE DANS L'ENSEIGNEMENT DES LANGUES ÉTRANGÈRES ? ÉTUDE DE CAS : PRZED SKLEPEM JUBILERA DE KAROL WOJTYŁA EN TRADUCTION L2
}

$$
\text { Streszczenie }
$$

Ces dernières années, le rôle de la traduction et des traductions dans l'enseignement des langues étrangères a été réévalué (Cook ; Laviosa ; Carreres, "Translation »). En 2001, le Cadre européen commun de référence (CECR) a reconnu la traduction et l'interprétation comme une activité linguistique appelée médiation. La traduction littéraire est proposée comme une activité de médiation, destinée à améliorer la compétence de médiation écrite des apprenants en langues étrangères. Cet article rend compte des résultats d'une étude de cas dans laquelle nous examinons dans quelle mesure la traduction de pièces de théâtre en L2 améliore en effet l'aptitude en matière de médiation des apprenants en langues. Dans cette étude de cas, nous avons décidé d'examiner dans quelle mesure les apprenants de néerlandais appliquent des stratégies de médiation lorsqu'ils traduisent un extrait de Przed sklepem jubilera en néerlandais (L2) et dans quelle mesure leurs stratégies diffèrent de celles que nous avons trouvées dans la traduction (officielle) en néerlandais de cette pièce de théâtre.

Mots-clé: didactique de la traduction, traduction pédagogique, stratégies de traduction, traduction littéraire.

\author{
TŁUMACZENIE LITERACKIE JAKO NARZĘDZIE DYDAKTYCZNE \\ W NAUCZANIU JEZYKÓW OBCYCH? \\ STUDIUM PRZYPADKU: PRZED SKLEPEM JUBILERA KAROLA WOJTYŁY \\ W TŁUMACZENIU L2 \\ Streszczenie
}

W ostatnich latach dokonano ponownej oceny roli tłumaczenia w nauczaniu języków obcych (Cook; Laviosa; Carreres, "Translation »). W 2001 r. Europejski System Opisu Kształcenia Językowego (ESOKJ) uznał tłumaczenie pisemne i ustne za działalność językową zwaną mediacją. Tłumaczenie literackie proponowane jest jako działanie mediacyjne, mające na celu poprawę umiejętności osób uczących się języków obcych w zakresie mediacji pisemnej. Niniejszy artykuł przedstawia wyniki studium przypadku, w którym badamy, w jakim stopniu tłumaczenie sztuki teatralnej w języku obcym rzeczywiście poprawia umiejętności mediacyjne osób uczących się języków obcych. W tym studium przypadku postanowiliśmy zbadać, w jakim stopniu osoby uczące się języka niderlandzkiego stosują strategie mediacyjne przy tłumaczeniu fragmentu sztuki Przed sklepem jubilera Karola Wojtyła na język niderlandzki (L2) i w jakim stopniu ich strategie różnią się od tych, które znaleźliśmy w (oficjalnym) niderlandzkim tłumaczeniu tej sztuki.

Słowa kluczowe: dydaktyka tłumaczenia; przekład edukacyjny; strategie thumaczeniowe; przekład literacki. 